\title{
Spatial Division Multiplexing for Optical Data Center Networks
}

\author{
Rui Lin ${ }^{1,5}$, Joris Van Kerrebrouck ${ }^{3}$, Xiaodan Pang ${ }^{1,4}$, Michiel Verplaetse ${ }^{3}$, Oskars Ozolins ${ }^{4}$, \\ Aleksejs Udalcovs ${ }^{4}$, Lu Zhang ${ }^{1}$, Lin Gan ${ }^{5}$, Ming Tang ${ }^{5}$, Songnian Fu ${ }^{5}$, Richard Schatz ${ }^{1}$, \\ Urban Westergren ${ }^{1}$, Sergei Popov ${ }^{1}$, Deming Liu ${ }^{5}$, Weijun Tong ${ }^{6}$, Timothy De Keulenaer ${ }^{7}$, \\ Guy Torfs ${ }^{3}$, Johan Bauwelinck ${ }^{3}$, Xin Yin ${ }^{3}$ and Jiajia Chen ${ }^{1,2}$ \\ ${ }^{1}$ KTH Royal Institute of Technology, Electrum 229, 16440 Kista, Sweden, \\ ${ }^{2}$ SCNU South China Normal University, \\ ${ }^{3}$ Ghent University - imec, IDLab, Department of Information Technology, Belgium \\ ${ }^{4}$ Networking and Transmission Laboratory, RISE Acreo AB, Kista, Sweden \\ ${ }^{5}$ Huazhong University of Science and Technology, Wuhan, China, tangming@ hust.edu.cn \\ ${ }^{6}$ Yangtze Optical fiber and Cable Joint Stock Limited Company (YOFC), Wuhan, China \\ ${ }^{7}$ BiFAST, spin-off of IDLab, Ghent University-imec, Ghent, Belgium \\ Email: jiajiac@kth.se
}

\begin{abstract}
Emerging mobile and cloud applications drive everincreasing capacity demands, particularly for short-reach optical communications, where low-cost and low-power solutions are highly required. Spatial division multiplexing (SDM) techniques provide a promising way to scale up the lane count per fiber, while reducing the number of fiber connections and patch cords, and hence simplifying cabling complexity. This talk will address challenges on both system and network levels, and report our recent development on SDM techniques for optical data center networks.
\end{abstract}

\section{INTRODUCTION}

O UURRENT demand for supporting data center applications has been posing stringent requirements on short-reach transmission techniques. To address the high capacity demand in data center networks (DCNs), scaling up the fiber capacity by spatial division multiplexing (SDM) approach has been proposed to boost the single fiber capacity, which can greatly reduce the number of fiber connections and patch cords and consequently, simplify cabling complexity [1]. Transporting data through different spatial channels in a single fiber, e.g., different modes in few mode fiber (FMF), independent cores in multicore fiber $(\mathrm{MCF})$ or a hybrid way, are widely studied[1][2]. In FMF based optical communications, complex digital signal processing (DSP) is needed to address the modal interference and differential mode dispersion, which hinders its deployment in DCNs. On the other hand, single mode (SM) MCF-based SDM system is appreciated for providing high capacity as well as good performance in DCNs, where low cost and low power transceivers are one of the main concerns.

Similarly, for the cost and complexity consideration in DCNs, intensity modulation and direct detection (IM/DD) systems are preferable for deployment. With the availability of

978-3-903176-07-2 @ 2018 IFIP high bandwidth optical transceivers [3], the simple non-to-zero (NRZ) and partial response modulation format, such as electrical duo-binary (EDB) are attractive for high-speed DCNs. With extremely low complex transceiver design, especially when the communications are achievable with analog equalization, real-time communication can be realized [4]. Many research efforts have been also put on advanced modulation formats, such as 4-level pulse amplitude modulation (PAM4) [5] and discrete multi-tone (DMT) [6] to support high-speed single lane transmission with relaxed requirement on the system bandwidth. For DCN applications for which distances of more than a few kilometres are required, recent works on high data rate advanced modulation signals transmission with $1.5-\mu \mathrm{m}$ single mode vertical cavity surface emitting laser (VCSEL) and single mode fiber (SMF) [6] also shows promising results to match the requirement on cost, energy consumption, footprint, and potential for seamlessly extension to long-term parallelism enabled by SDM techniques.

In this paper, we report our recent work [7-9] to address the high-speed requirement in SDM-enabled DCNs. Using 7-core fiber, the demonstration of i) real-time $100 \mathrm{Gbps} / \lambda /$ core NRZ and EDB transmission over $10 \mathrm{~km} \mathrm{MCF}$ with optical dispersion compensation with BiCMOS based tranmistter and receiver chip and a monolithic C-band $100 \mathrm{GHz}$ distributed feedback electro-absorption modulated laser (DFB-EAM) [7]; with a 1.5$\mu \mathrm{m}$ SM-VCSEL ii) $50 \mathrm{Gbaud} / \lambda /$ core PAM-4 over $1 \mathrm{~km}$ dispersion-uncompensated and $10 \mathrm{~km}$ dispersion-compensated MCF links [8]; and iii) total net rate over 700 Gbps DMT optical signals over $2.5 \mathrm{~km}$ dispersion-uncompensated MCF [9]. In all of the demonstrations above, bit error rate (BER) below the 7\%overhead hard decision forward error correction code limit (7\%-OH HD-FEC) is achieved.

\section{EXPERIMENTAL SETUP}

The experiment setup of the $100 \mathrm{Gbps} / \lambda /$ core demonstration of real-time NRZ/EDB and VCSEL-based PAM4 and DMT transmission is illustrated in Fig.1. For the NRZ and EDB signal 

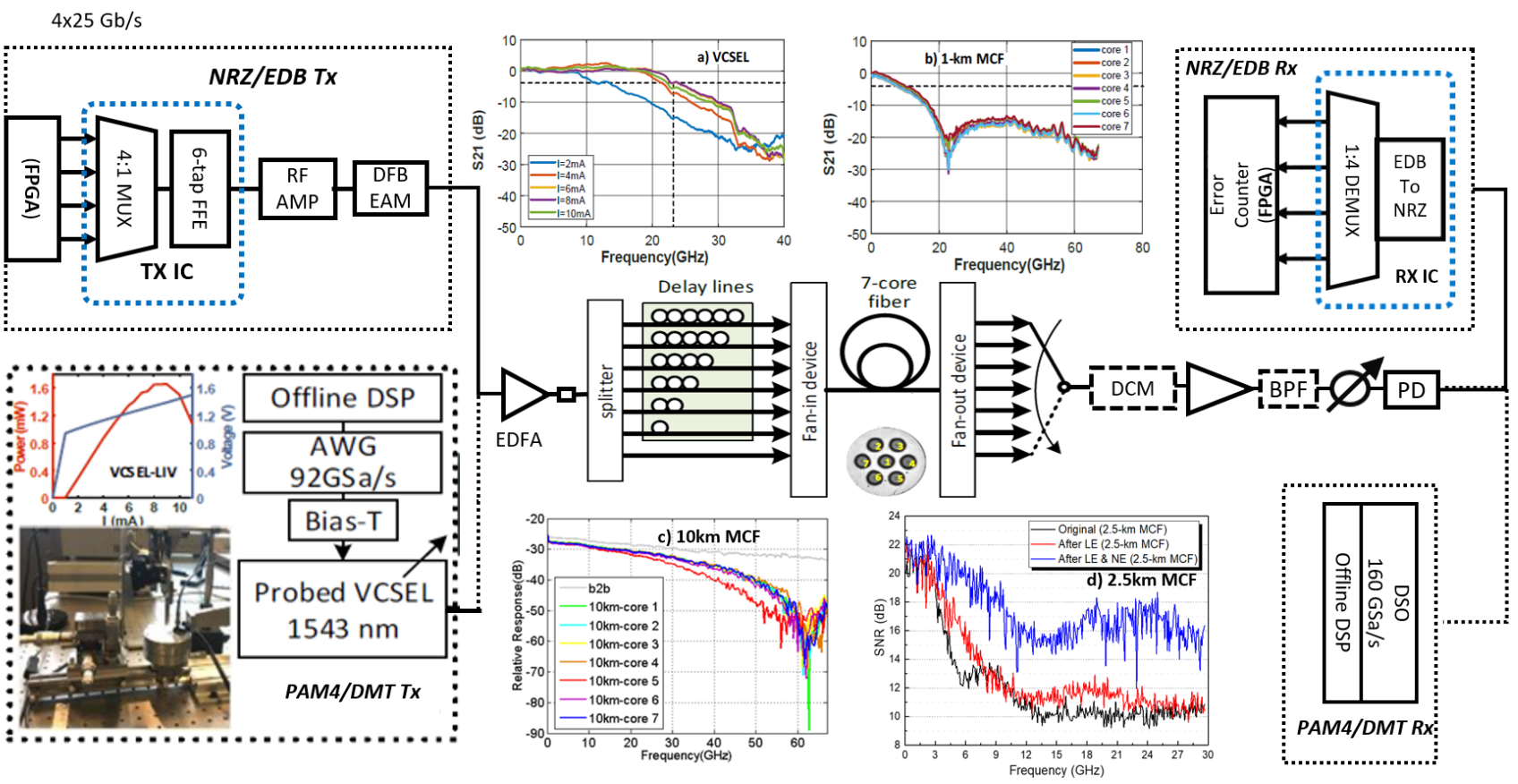

Fig. 1 The experimental setup of the high-speed transmission based on MCF enabled SDM systems for optical interconnects.

generation, four electrical $2^{7}-1$ pseudo-random bit streams (PRBS) at 25 Gbps is generated by a Xilinx Virtex Ultrascale FPGA and multiplexed into a 100 Gbps NRZ signal by the transmitter (TX) IC. A six-tap analog feedforward equalizer (FFE) at the TX side is used to compensate the frequency rolloff and dispersion induced inter-symbol-interference in the link. A $50 \mathrm{GHz} \mathrm{RF}$ amplifier is used to drive a C-band $100 \mathrm{GHz}$ distributed feedback electro-absorption modulated laser (DFBEAM) [3]. The DFB-EAM is driven by $117.0 \mathrm{~mA}$ and biased at $-1.85 \mathrm{~V}$, emits at $1548.7 \mathrm{~nm}$.

The advanced modulation formats, i.e., PAM4 and DMT signals are generated offline. Specifically, the PAM-4 symbols are offline generated, before being up-sampled and filtered with a raise cosine filter of 0.15 roll-off factor. Based on the characterized end-to-end channel frequency response preequalization is performed. In the DMT signal generation the length of the inverse fast Fourier transform (IFFT) and cyclic prefix were set to 1024 and 16 and the first subcarrier is set to null. The linear channel equalization (LE) pilot ratio was 3.3\%. Volterra nonlinear equalization (NLE) was used to compensate the VCSEL. Clipping ratio is set to 0.7 to improve the output signal to noise ratio (SNR) from the DAC. The VCSEL is operated at room temperature without active cooling, and the optimal driving current is found to be $7.8 \mathrm{~mA}$, considering both bandwidth and output power.

The generated signals from the transmitters are amplified and split into 7 branches using a $1 \times 8$ splitter and further decorrelated with different delays and launched into the 7 cores of the MCF via a low-loss and low crosstalk fan-in (FI)/fanout(FO). The FI/FO module is fusion spliced to the MCF at one end, split and connected to seven individual single mode fiber (SMF) pigtails at the other end. The frequency response of the link in back to back (b2b) configuration and MCF transmission in different lengths, i.e., $1 \mathrm{~km}, 2.5 \mathrm{~km}$ and $10 \mathrm{~km}$ can be found in Fig.1. In the case of $10 \mathrm{~km} \mathrm{MCF}$, a fixed dispersion compensation module (DCM) of $-159 \mathrm{ps} / \mathrm{nm}$ is used for coarse dispersion compensation. Approximate homogeneous characteristics of the cores can be found due to small difference between the responses. It should be noted that, in the VCSELMCF link the mux and demux process in practice should be more effectively achieved with a VCSEL/PD array buttcoupled to each core of the MCF.

At the receiver side, an EDFA and a variant optical attenuator (VOA) are used to adjust the received optical power. The optical signal is detected by an InP-based PIN-PD (>90 GHz 3$\mathrm{dB} B W$ ) with a responsivity of $0.2 \mathrm{~A} / \mathrm{W}$. The received $100 \mathrm{Gbps}$ data is deserialized by the receiver (RX) IC into 4x25 Gbps streams for real-time error detection. For receiving PAM4 and DMT signal detection, offline DSP is carried out, while for the case of NRZ/EDB real-time transmission is implemented.

\section{RESULTS AND ANALYSIS}

In both DFB-EAM and VCSEL based SDM systems, 7\%$\mathrm{OH}$ HD-FEC is reached for all the investigated modulation schemes. In particular, some of the performance evaluation results can be found in Fig. 2, the performance of the received EDB after $10 \mathrm{~km}$ MCF transmission is shown in Fig. 2(a). Thanks to the broadband optical transceiver, BER $=2.8 \mathrm{E}-6$ is reached for both NRZ and EDB signals with the received power of around $7 \mathrm{dBm}$ in b2b configuration. Except for core 5, all the other spatial channels have similar receiver sensitivity to reach the $\mathrm{BER}=3.8 \mathrm{E}-3$ after $10 \mathrm{~km}$ MCF transmission. The sensitivity variation is mainly due to the end face reflection in the MCF link. With higher received optical power, in which all the spatial channels are well below the KP4 FEC limit (BER=2.0E-4).

Fig. 2(b) shows the BER performance after the $1 \mathrm{~km} \mathrm{MCF}$ 


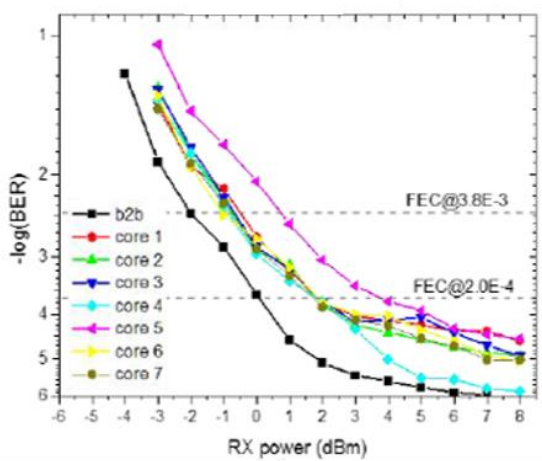

(a)

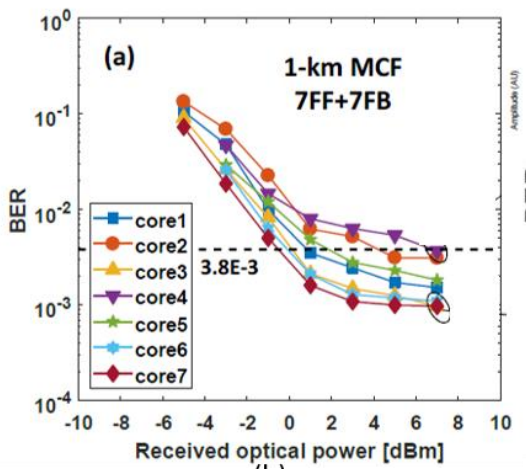

(b)

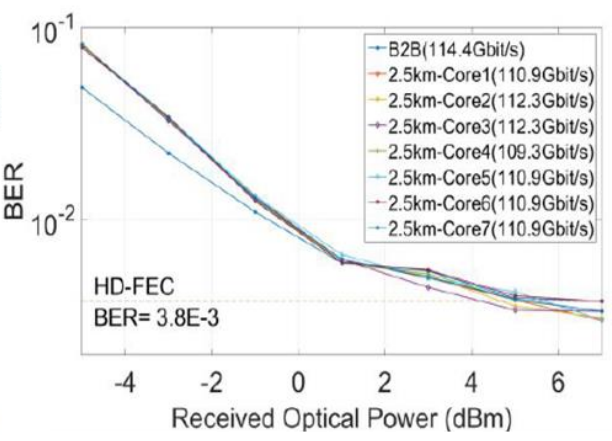

(c)

Fig. 2 BER performance of (a) 100Gbps EDB transmission over $10 \mathrm{~km}$ dispersion compensated MCF, (b) 50 Gbaud PAM-4 signal after $1 \mathrm{~km}$, and (c) the DMT signals transmission over $2.5 \mathrm{~km} \mathrm{MCF}$.

transmissions for PAM-4 signal at 50 Gbaud. For the uncompensated $1 \mathrm{~km}$ MCF cases, a DFE of 7-feed forward (FF)-tap + 7-feedback (FB)-tap can successfully recover the received signals in all the cores to reach BER performance below the 7\%-OH-HD-FEC limit. Additionally, different performances between different cores can be seen which is in accordance with the differences in the characterized frequency response. It should be noted that due to added complexity, we did not apply any specific amplitude-level dependent symbol decision method or nonlinear equalization techniques to mitigate the eye skew. Therefore, due to narrower horizontal eye opening, better BER performance and/or higher baud rates can be potentially achieved after eye skew correction.

As for the DMT case, the measured BER in function of the received optical power (RoP) at the PD input is shown in Fig. 2(c). The 7\% HD-FEC limit is achieved with NLE. The achieved gross bit rate is $114.4 \mathrm{~Gb} / \mathrm{s}$ for the B2B case. For 2.5 $\mathrm{km}$ MCF transmission, we have achieved gross-rates of 110.9Gb/s, 112.3-Gb/s, 112.3-Gb/s, 109.3-Gb/s, 110.9-Gb/s, 110.9$\mathrm{Gb} / \mathrm{s}$, and $110.9-\mathrm{Gb} / \mathrm{s}$, respectively. The total system capacity with $2.5-\mathrm{km} \mathrm{MCF}$ is about $777.5 \mathrm{~Gb} / \mathrm{s}$ (net rate $726.7 \mathrm{~Gb} / \mathrm{s}$ ).

\section{CONCLUSIONS}

Based on the MCF enabled SDM, the IM/DD $7 \times 100 \mathrm{Gbps} / \lambda /$ core communication and beyond are achieved. Real-time NRZ/EDB transmission are realized with monolithic DFB-EAM and BiCMOS based transceiver chips while highspeed PAM4 and DMT signal transmission are carried out with 1.5 um single mode-VCSEL. The presented SDM system proves its potential in providing high-speed, low cost, and low power optical interconnect for DCNs.

\section{ACKNOWLEDGMENT}

This work was supported by the National Natural Science Foundation of China (61331010, 61722108, 61775137, 61671212, and 61550110240), Swedish Research Council Swedish Research Council projects PHASE (2016-04510) and Go-iData (2016-04489), the Swedish Foundation for Strategic Research (SSF), the Göran Gustafsson Foundation, the Swedish ICT-TNG, the Celtic-Plus sub-project SENDATE-EXTEND \& SENDATE FICUS funded by Vinnova, the Flemish FWO \&
IWT, IOF, EU H2020 projects STREAMS (688172), TeraBoard (688510), PICTURE (780930), and the Natural Science Foundation of Guangdong Province.

\section{REFERENCES}

[1] R. G. H. van Uden, et al., Ultra-high-density spatial division multiplexing with a few-mode multicore fibre, Nat. Photonics. 8 (2014) 865-870.

[2] D. J. Richardson, et al., Space-division multiplexing in optical fibres Nat. Photonics. 7 (2013) 354.

[3] M. Chaciński, et al., Monolithically integrated $100 \mathrm{GHz}$ DFB-TWEAM, J. Light. Technol. 27 (2009) 3410-3415.

[4] M. Verplaetse, et al., Real-time $100 \mathrm{~Gb} / \mathrm{s}$ transmission using three-level electrical duobinary modulation for shortreach optical interconnects, J. Light. Technol. 35 (2017) 13131319.

[5] J. Verbist, et al., DAC-less and DSP-free PAM-4 transmitter at $112 \mathrm{~Gb} / \mathrm{s}$ with two parallel GeSi electroabsorption modulators, in ECOC PDP 2017.

[6] C. Kottke, et al., High speed $160 \mathrm{~Gb} / \mathrm{s}$ DMT VCSEL transmission using pre-equalization, in OFC.2017.

[7] R. Lin, et al., Real-time $100 \mathrm{Gbps} / \lambda$ /core NRZ and EDB IM/DD transmission over $10 \mathrm{~km}$ multicore fiber, in OFC, 2018. [8] X. Pang, et al., $7 \times 100$ Gbps PAM-4 transmission over 1$\mathrm{km}$ and 10-km single mode 7-core fiber using 1.5- $\mu \mathrm{m}$ SMVCSEL, in OFC, 2018.

[9] J. Van Kerrebrouck, et al.,Single-mode VCSEL discrete multi-tone transmission over 2.5 -km multicore fiber, in OFC, 2018. 